\title{
Magneto-Rotational Symmetry in Chiral Magnetoelectrodeposition
}

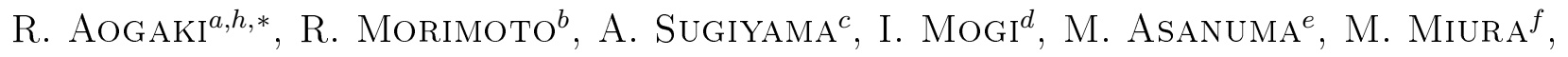 \\ Y. OSHIKIRI ${ }^{g}$, Y. YAMAUCHI ${ }^{h}$ \\ ${ }^{a}$ Polytechnic University, 2-20-12-1304, Ryogoku, Sumida, Tokyo 130-0026, Japan \\ ${ }^{b}$ Saitama Prefectural Okubo Water Filtration Plant, Saitama 338-0814, Japan \\ ${ }^{c}$ Waseda University, Shinjuku, Tokyo 169-0855, Japan \\ ${ }^{d}$ IMR, Tohoku University, Katahira, Aoba-ku, Sendai 980-8577, Japan \\ eYokohama Harbour Polytechnic College, Yokohama 231-0811, Japan \\ ${ }^{f}$ Polytechnic College Akita, Odate, Akita 017-0805, Japan \\ ${ }^{g}$ Yamagata College of Industry and Technology, Yamagata 990-2473, Japan \\ ${ }^{h}$ National Institute for Materials Science, Tsukuba, Ibaraki 305-0044, Japan
}

\begin{abstract}
The chiral activities of electrodeposited surfaces fabricated under vertical magnetic fields were theoretically examined. Chiral activities arise from the micro-vortexes, called micro-MHD (magnetohydrodynamic) flows (MMF), activated under a tornado-like vortex called vertical MHD flow (VMF). It was concluded that the interaction between MMF and VMF determines the activities.
\end{abstract}

DOI: $10.12693 /$ APhysPolA.126.378

PACS: 47.35.Tv

\section{Introduction}

Using electrodes fabricated by magnetoelectrodeposition, Mogi has first found the appearance of chiral activity in enantiomorphic electrode reactions, which was thought to come from screw dislocation formed by vortex flows $[1,2]$. In a vertical magnetic

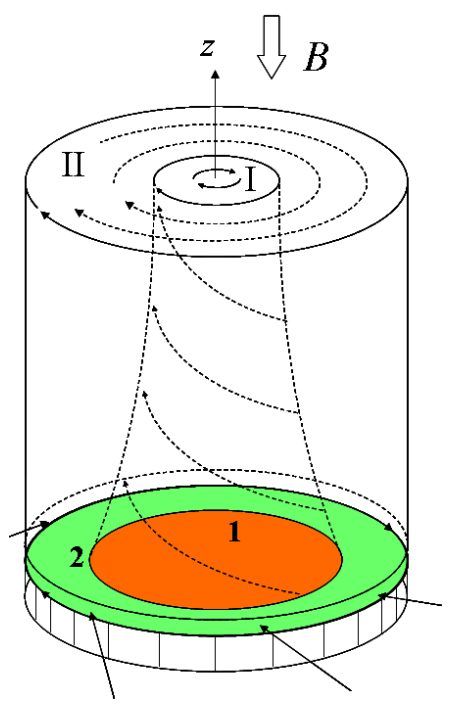

Fig. 1. Vertical MHD flow.

*corresponding author; e-mail: aogaki.ryoichi@nims.go.jp field, as shown in Fig. 1, a tornado-like rotating stream (VMF) emerges over the electrode surface. Inside the stream, micro-vortexes (MMF) are activated. The chirality of the screw dislocation is thus ascribed to that of MMF, which is in turn determined by the directions of magnetic field and VMF rotation. The purpose of the present paper is to report the result of the theoretical analysis on the chiral activities produced by magnetic field and VMF rotation.

\section{Theory}

In electrodeposition, due to friction, an ordinal rigid electrode surface provides stationary diffusion layer, which yields deposition irrelevant to vortex flow. For the transcription of the vortex motion to the deposit surface to make a free surface without friction, something like atomic-scale lubricant is required. Recently, it has been found that ionic vacancy is created by electrode reaction, acting as a lubricant [3-5]. In consequence, two types of surfaces are self-organized on the electrode surface; one is ordinal rigid surface with friction, and the other is free surface without friction covered with ionic vacancies. At the same time, two types of MMF with different flow and rotational directions are activated on the rigid and free surfaces, respectively. The rotational directions of these vortexes are determined by the direction of magnetic field, whereas the rotation of the VMF donates the precessions to them. Figure 2 represents the schematic figure of the two kinds of MMF activated by magnetic field, the flow modes of which are classified into four types, i.e., upward or downward and clockwise $(\mathrm{CW})$ or anticlockwise $(\mathrm{ACW})$. For simplicity, 
assuming that magnetic field is directed downward, we have upward $\mathrm{ACW}$ and downward $\mathrm{CW}$ flows activated on the free and rigid surfaces, respectively. As a result, on the electrode surface, an activated MMF layer is first formed. Then, due to momentum conservation, the second layer symmetrically involving other MMF's emerges on the first lower MMF layer, CW rotating together with the VMF, so that the MMF's in the second upper layer receive the Coriolis force from the VMF rotation, which, as is well-known in meteorology, donates ACW and $\mathrm{CW}$ rotational forces to the streams at the outlet and the inlet, respectively. As shown in Fig. 2, the upward flow

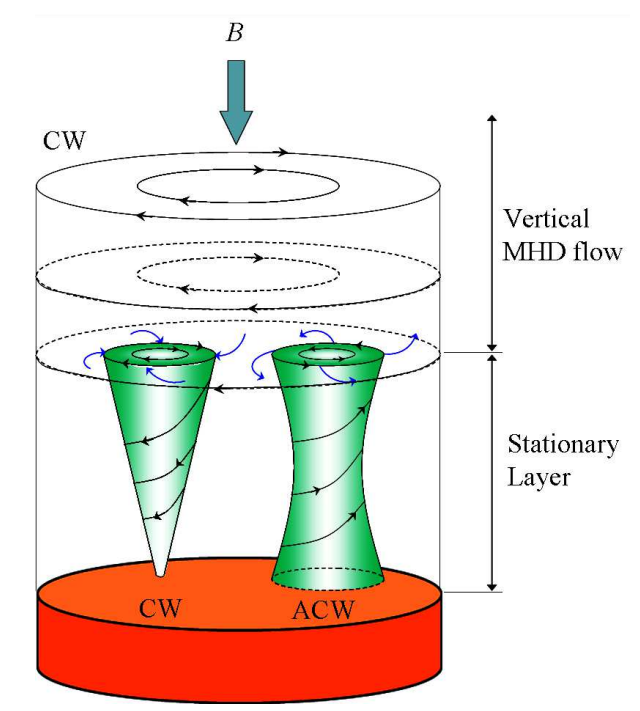

Fig. 2. Micro-MHD flows.

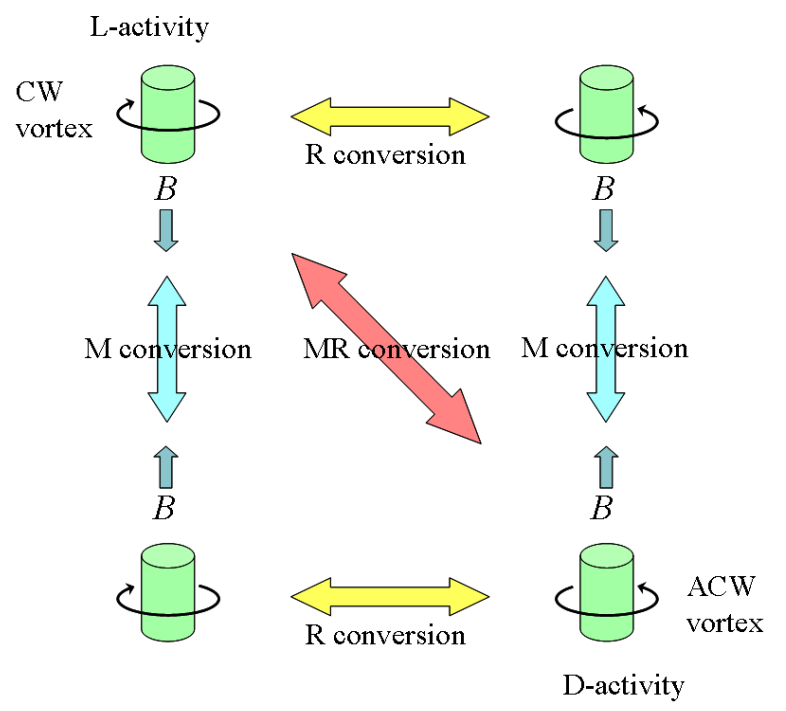

Fig. 3. Magneto-rotational symmetry. in the lower layer is gushed from the outlet in the upper layer, whereas the downward flow in the lower layer is drained into the inlet in the upper layer, so that the upward and downward flows in the lower layer receive the $\mathrm{ACW}$ and $\mathrm{CW}$ Coriolis forces from the upper layer, respectively. Such directions are consistent with those of the initially activated MMF's, so that their rotations are accelerated, yielding stable ACW and CW MMF's. However, since the atomic-scale transcription is possible on the free surface, only ACW motion is transcribed to the deposit surface. Due to the step growth toward the upstream side, a screw dislocation develops in the opposite $(\mathrm{CW})$ direction. In view of the mirror-image relationship between chiral catalyst and enantiomeric reagent, it is concluded that the deposit surface under a downwardoriented magnetic field acquires L-activity corresponding to the $\mathrm{CW}$ dislocation. On the contrary, in an upwardoriented magnetic field, a D-active surface is predicted (Fig. 3).

\section{Conclusion}

It was theoretically predicted that under downwardand upward-oriented magnetic fields, L-active and Dactive electrodes are fabricated, respectively. These theoretical predictions were consistent with the experimental results reported by Mogi.

\section{Acknowledgments}

The authors thank the Tsukuba Magnet Laboratory, National Institute for Materials Science (NIMS) and the High Field Laboratory for Superconducting Materials, Institute for Materials Research (IMR), Tohoku University (Project No 12H0027) for financial support and access to superconducting magnets.

\section{References}

[1] I. Mogi, K. Watanabe, Int. J. Electrochem. 2011, ID 239637 (2011).

[2] I. Mogi, R. Morimoto, R. Aogaki, K. Watanabe, Sci. Rep. 3, 2574 (2013).

[3] AR. Aogaki, Electrochem. 76, 458 (2008).

[4] R. Aogaki, K. Motomura, R. Morimoto, A. Sugiyama, I. Mogi, M. Asanuma, M. Miura, Y. Oshikiri, Y. Yamauchi, ECS Trans. 45, 9 (2013).

[5] A. Sugiyama, R. Aogaki, R. Morimoto, M. Miura, Y. Oshikiri, M. Miura, I. Mogi, T. Osaka, Electrochem. 81, 890 (2013). 